\title{
Guest editorial for the IJCARS special issue on MICCAI 2017
}

\author{
Maxime Descoteaux ${ }^{1} \cdot$ Lena Maier-Hein ${ }^{2} \cdot$ Alfred Franz $^{3} \cdot$ Pierre Jannin $^{4} \cdot$ Louis D. Collins $^{5} \cdot$ Simon Duchesne $^{6}$
}

Published online: 17 August 2018

c) CARS 2018

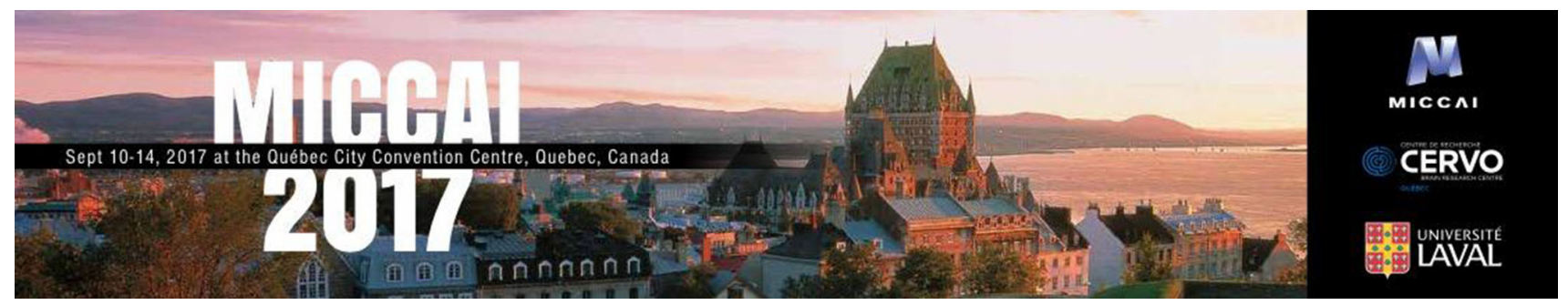

We were very proud to host the 20th Medical Image Computing and Computer Assisted Interventions (MICCAI) conference at the Quebec City Conference Center from September 10 to 14, 2017, in Quebec City, Canada. Ce

Maxime Descoteaux

maxime.descoteaux@usherbrooke.ca

$\bowtie$ Lena Maier-Hein

1.maier-hein@dkfz-heidelberg.de

Alfred Franz

franz@hs-ulm.de

Pierre Jannin

pierre.jannin@univ-rennes1.fr

Louis D. Collins

louis.collins@mcgill.ca

Simon Duchesne

simon.duchesne@fmed.ulaval.ca

1 Computer Science, Université de Sherbrooke, 2500 Boul. Université, Sherbrooke, QC J1K2R1, Canada

2 Computer-Assisted Medical Interventions, German Cancer Research Center (DKFZ), Im Neuenheimer Feld 581, 69120 Heidelberg, Germany

3 Department of Computer Science, Ulm University of Applied Sciences, Albert-Einstein-Allee 55, 89081 Ulm, Germany

4 Modeling Surgical Knowledge and Processes, U1099 INSERM Rennes, Faculté de Médecine, Université de Rennes 1, CS34317, 35043 Rennes cedex, France

5 Neurology and Neurosurgery, Biomedical Engineering, McGill University, 3801 University Street, Montreal, QC H3A 2B4, Canada

6 Radiology Department, Université Laval, 2601 Chemin de la Canardière, Québec, QC G1J 2G3, Canada fut un plaisir et une fierté de vous recevoir tous et chacun à Québec, berceau de la culture francophone en Amérique du Nord. MICCAI 2017 was organized out of Université Laval (Quebec City, Canada) in collaboration with the German Cancer Research Center (Heidelberg, Germany); McGill University (Montréal, Canada); Université de Rennes I (Rennes, France); and Université de Sherbrooke (Sherbrooke, Canada). The MICCAI 2017 conference attracted over 1360 world's leading scientists, engineers, and clinicians, involved in medical image processing, medical image formation, and computer-assisted medical procedures.

We received 800 submissions to our call for papers, which were reviewed by the Program Committee, composed of 53 domain experts, alongside $>600$ reviewers from the community, following an updated and rigorous double-blind review process. Ultimately, 255 manuscripts were accepted, with 45 accepted papers (18\%) first authored by female scientists (out of 164 papers submitted by a woman as first author).

These 255 papers touched on 15 important thematic groups, namely (number of manuscripts in parentheses): Machine Learning in Medical Imaging Computing (56), Feature Extraction and Classification Techniques (23), Diffusion Magnetic Resonance Imaging (MRI) and Tensor/Fiber Processing (20), Optical Imaging (18), Functional Imaging, Connectivity and Brain Parcellation (17), Motion and Cardiac Analysis (16), Registration Techniques (16), Atlas and Surface-Based Techniques (14), Interventional Imaging and Navigation (14), Image Segmentation and Modelling (12), Shape and Patch-based Techniques (11), Planning and Simulation for Medical Interventions (11), Airway an Vessel 
Analysis (10), Tumor Processing (9), and Medical Image Computing (8).

Overall, the authors of seven selected papers followed our invitation for publication in this Special Issue of the International Journal of Computer Assisted Radiology and Surgery (IJCARS). The expanded manuscripts underwent the regular IJCARS review process and met both its high standard for acceptance and deadline for publication. Four papers are included in this issue.

The manuscript "A radiation-free mixed-reality training environment and assessment concept for C-arm-based surgery" proposes a hybrid simulation system for training C-Arm imaging-based surgical procedures. Preliminary evaluation was performed for spinal surgery and showed accuracy of the system, internal validity, and positive user acceptability.

The paper "Surgical Soundtracks: Towards Automatic Musical Augmentation of Surgical Procedures" was awarded one of the MICCAI young investigator awards. It addresses the problem that visual feedback has traditionally been the dominant way of conveying information in the operating room (OR), leading to cognitive overload. The work presents a highly innovative approach to auditory augmentation which is based on the automatic generation of pleasant and ergonomic audio in complex routines, as opposed to overly simplistic feedback, to avoid fatigue.
The manuscript "TernaryNet: Faster Deep Model Inference without GPUs for Medical 3D Segmentation using Sparse and Binary Convolutions" introduces a method for reducing memory requirements and computation time not based on GPU. It is demonstrated for pancreas segmentation on CT images without significant accuracy decrease.

The paper "Robust and semantic needle detection in 3D ultrasound using orthogonal-plane convolutional neural networks" proposes an original approach for automatic needle detection and segmentation in 3D ultrasound images. With experiments in animal images, they show promising results demonstrating the feasibility of such approach.

In closing, we would like to thank all members of the Program Committee as well as the reviewers for their support during the entire MICCAI 2017 and IJCARS Special Issue process. Last but not least, we thank all authors, co-authors, students, and supervisors, who toiled away to produce work of exceptional quality that maintains MICCAI as a beacon of savoir-faire and expertise not to be missed. We feel confident that this Special Issue provides an interesting window into this body of work and will entice readers to continue their association with, or join anew the MICCAI community.

\section{Compliance with ethical standards}

Conflict of interest The authors declare that they have no conflict of interest. 